\title{
Simplifying the tree-level superstring massless five-point amplitude
}

\author{
Carlos R. Mafra \\ Max-Planck-Institut für Gravitationsphysik, Albert-Einstein-Institut, \\ 14476 Golm, Germany \\ E-mail: crmafra@aei.mpg.de
}

ABSTRACT: We use the pure spinor formalism to obtain the supersymmetric massless fivepoint amplitude at tree-level in a streamlined fashion. We also prove the equivalence of an OPE identity in string theory with a subset of the Bern-Carrasco-Johansson five-point kinematic relations, and demonstrate how the remaining BCJ identities follow from the different integration regions over the open string world-sheet, therefore providing a first principles derivation of the (supersymmetric) BCJ identities.

KEYWORDS: Superstrings and Heterotic Strings, Superspaces

ARXIV EPRINT: 0909.5206 


\section{Contents}

1 Introduction 1

2 The five point amplitude in pure spinor superspace 2

3 Derivation of the BCJ kinematic identities 5

$\begin{array}{ll}\text { A Computation of the kinematic factors } & \mathbf{1 0}\end{array}$

A.1 Explicit proof of $L_{2331}=L_{3121}-L_{2131}$

B A different pure spinor superspace expression for $A_{F^{4}}$

$\begin{array}{ll}\text { C The MHV amplitude } & 14\end{array}$

$\begin{array}{ll}\text { D The integrals } K_{j} & 15\end{array}$

\section{Introduction}

Since the discovery of the pure spinor formalism [1] a new method to efficiently compute supersymmetric scattering amplitudes is available. Although its simplifying features manifest themselves more vividly in explicit one- and two-loop computations [2-6] and provide hope $^{1}$ for higher-loop extensions [10-12], tree-level amplitudes [13] also benefit from the streamlined nature of the formalism. ${ }^{2}$ In particular, having results written in terms of pure spinor superspace expressions $[6,15]$ sheds new light into finding supersymmetric completions [16].

This paper simplifies the long (bosonic) RNS five-point computations of $[17,18]$ while naturally extending them to the full supersymmetric multiplet using the pure spinor superspace. In doing that we uncover the superstring origin of the Bern-Carrasco-Johansson (BCJ) kinematic identities of [19], proving that some of them come from an OPE identity and that they are supersymmetric. And in view of the string theory proof for the fourpoint BCJ identity we will demonstrate that the remaining BCJ relations follow from the different integration regions of the open string world-sheet. These integrations over the various domain of integrations are related to the monodromy identities [20,21] between the string theory amplitudes which have been used in [20] to prove that the number of partial amplitudes is $(N-3)$ !, and is ultimately related to the BCJ identities of [19].

\footnotetext{
${ }^{1}$ Useful knowledge can be obtained even without fully explicit higher-loop computations [7-9].

${ }^{2}$ For a review of scattering amplitudes in the pure spinor formalism, see [14].
} 
In the following, the open string massless five-point amplitude at tree-level will be shown to be $\mathcal{A}_{5}=\sum_{\text {non-cyclic }} \operatorname{tr}\left(\lambda^{a_{i_{1}}} \ldots \lambda^{a_{i_{5}}}\right) A\left(i_{1}, \ldots, i_{5}\right)$, with

$$
A(1,2,3,4,5)=\frac{\tilde{L}_{2131}}{\alpha_{12} \alpha_{45}} S_{1}-\frac{\tilde{L}_{2334}}{\alpha_{23} \alpha_{51}} S_{2}-\frac{\tilde{L}_{2134}}{\alpha_{12} \alpha_{34}} S_{3}-\frac{\tilde{L}_{2331}}{\alpha_{23} \alpha_{45}} S_{4}-\frac{\tilde{L}_{3424}}{\alpha_{34} \alpha_{51}} S_{5}+L_{2431} K_{3}\left(2 \alpha^{\prime}\right)^{2}
$$

where

$$
\begin{aligned}
& S_{1}=T-\left(2 \alpha^{\prime}\right)^{2} K_{3}\left(\alpha_{34} \alpha_{45}+\alpha_{23} \alpha_{12}\right), \\
& S_{2}=T-\left(2 \alpha^{\prime}\right)^{2} K_{3}\left(\alpha_{12} \alpha_{23}+\alpha_{51}\left(\alpha_{45}+\alpha_{23}\right)\right) \\
& S_{3}=T-\left(2 \alpha^{\prime}\right)^{2} K_{3}\left(\alpha_{34} \alpha_{45}+\alpha_{12}\left(\alpha_{51}+\alpha_{34}\right)\right), \\
& S_{4}=T-\left(2 \alpha^{\prime}\right)^{2} K_{3}\left(\alpha_{12} \alpha_{23}+\alpha_{45}\left(\alpha_{51}+\alpha_{23}\right)\right) \\
& S_{5}=T-\left(2 \alpha^{\prime}\right)^{2} K_{3}\left(\alpha_{12} \alpha_{51}+\alpha_{23} \alpha_{34}\right),
\end{aligned}
$$

and $T=1+O\left(k^{6}\right)+\ldots$ and $K_{3}=\zeta(2)+O\left(k^{2}\right)+\ldots$ have well-known expansions in terms of $\alpha_{i j}=\left(k^{i} \cdot k^{j}\right)$ [18]. In the field theory limit we set $\alpha^{\prime} \rightarrow 0$ and therefore $S_{j} \rightarrow 1$. The kinematic factors $\tilde{L}_{i j k l}$ are given by simple pure spinor superspace expressions which satisfy the supersymmetric BCJ relations,

$$
\tilde{L}_{2331}=L_{3121}-\tilde{L}_{2131}, \quad \tilde{L}_{2334}=\tilde{L}_{3424}-L_{2434} .
$$

The paper is organized as follows. In section 2 we compute the five-point amplitude at tree-level and express it in terms of simple pure spinor superspace expressions. In section 3 we prove an OPE identity from which the supersymmetric generalizations for some of the BCJ relations can be obtained. Furthermore, using the analogy with the four-point amplitude derivation of the BCJ identity we show how to obtain the remaining ones. The pure spinor superspace computations are presented in appendix A, together with the explicit proof of (1.2) directly in superspace. Of particular importance is the simplified expression for the OPE of two integrated vertices presented in (A.2). The appendix B is devoted to writing down an ansatz for a simplified expression of $A_{F^{4}}(\theta)$, whose bosonic component expansion agrees with the expression obtained in section 2 . The appendix $\mathrm{C}$ is a formal rewriting of the ten-dimensional results using the four-dimensional spinor helicity formalism, and we show agreement with the expressions of [22-25]. Finally, in appendix D we derive the relations obeyed by the integrals $K_{j}$ which were used in section 2 .

\section{The five point amplitude in pure spinor superspace}

Following the tree-level prescription of [1] the open superstring 5-point amplitude is

$$
\mathcal{A}_{5}=\sum_{\text {non-cyclic }} \operatorname{tr}\left(\lambda^{a_{i_{1}}} \ldots \lambda^{a_{i_{5}}}\right) A\left(i_{1}, \ldots, i_{5}\right)
$$

where the partial amplitude $A_{5}(1,2,3,4,5)$ is given by

$$
A_{5}(1,2,3,4,5)=\left\langle\left(\lambda A^{1}\right)\left(z_{1}\right)\left(\lambda A^{4}\right)\left(z_{4}\right)\left(\lambda A^{5}\right)\left(z_{5}\right) \int d z_{2} U^{2}\left(z_{2}\right) \int d z_{3} U^{3}\left(z_{3}\right)\right\rangle .
$$


The $\mathrm{SL}(2, R)$ symmetry of the disc requires the fixing of three positions, chosen as $\left(z_{1}, z_{4}, z_{5}\right)=(0,1, \infty)$. Therefore the integrals are over the region $0 \leq z_{2} \leq z_{3} \leq 1$.

Using the OPEs of the pure spinor formalism to integrate out the conformal weight-one variables, (2.1) assumes the following form

$A_{5}=\int d z_{2} d z_{3} \prod_{i<j}\left|z_{i j}\right|^{2 \alpha^{\prime} k_{i} \cdot k_{j}}\left[\frac{L_{2131}}{z_{21} z_{31}}+\frac{L_{2134}}{z_{21} z_{34}}-\frac{L_{2434}}{z_{24} z_{34}}-\frac{L_{2431}}{z_{24} z_{31}}+\frac{L_{2331}}{z_{23} z_{31}}-\frac{L_{2334}}{z_{23} z_{34}}+\frac{L_{2314}}{z_{23}^{2}}\right]$

where the kinematic factors $L_{i j k l}$ are given by the following pure spinor superspace expressions (from now on we set $2 \alpha^{\prime}=1$ ),

$$
\begin{aligned}
L_{2134}= & \left\langle\left[A_{m}^{1}\left(\lambda \gamma^{m} W^{2}\right)+\left(\lambda A^{1}\right)\left(k^{1} \cdot A^{2}\right)\right]\left[A_{n}^{4}\left(\lambda \gamma^{n} W^{3}\right)+\left(\lambda A^{4}\right)\left(k^{4} \cdot A^{3}\right)\right]\left(\lambda A^{5}\right)\right\rangle \\
& +\left\langle\left(k^{1} \cdot k^{2}\right)\left(A^{4} W^{3}\right)\left(\lambda A^{1}\right)\left(\lambda A^{2}\right)\left(\lambda A^{5}\right)\right\rangle+\left\langle\left(k^{3} \cdot k^{4}\right)\left(A^{1} W^{2}\right)\left(\lambda A^{3}\right)\left(\lambda A^{4}\right)\left(\lambda A^{5}\right)\right\rangle \\
L_{2131}= & +\left\langle\left[A_{m}^{1}\left(\lambda \gamma^{m} W^{2}\right)+\left(\lambda A^{1}\right)\left(k^{1} \cdot A^{2}\right)\right]\left(\lambda A^{4}\right)\left(\lambda A^{5}\right)\left(\left(k^{1}+k^{2}\right) \cdot A^{3}\right)\right\rangle \\
& +\left\langle\left[A_{m}^{1}\left(\lambda \gamma^{m} W^{3}\right)\left(k^{1} \cdot A^{2}\right)-A^{1 m}\left(\lambda \gamma^{n} W^{3}\right) \mathcal{F}_{m n}^{2}-\left(\lambda \gamma^{m} W^{3}\right)\left(W^{1} \gamma^{m} W^{2}\right)\right]\left(\lambda A^{4}\right)\left(\lambda A^{5}\right)\right\rangle \\
& +\left\langle\left[\left(k^{1} \cdot k^{2}\right)\left(A^{1} W^{3}\right)\left(\lambda A^{2}\right)-\left(k^{1} \cdot k^{2}\right)\left(A^{2} W^{3}\right)\left(\lambda A^{1}\right)\right]\left(\lambda A^{4}\right)\left(\lambda A^{5}\right)\right\rangle \\
& +\left\langle\left[\left(k^{1} \cdot k^{3}\right)\left(A^{1} W^{2}\right)\left(\lambda A^{3}\right)+\left(k^{2} \cdot k^{3}\right)\left(A^{1} W^{2}\right)\left(\lambda A^{3}\right)\right]\left(\lambda A^{4}\right)\left(\lambda A^{5}\right)\right\rangle \\
L_{2331}= & \left\langle\left[A_{m}^{1}\left(\lambda \gamma^{m} W^{3}\right)\left(k^{3} \cdot A^{2}\right)+\frac{1}{4}\left(\lambda \gamma^{p} \gamma^{m n} W^{3}\right) A_{p}^{1} \mathcal{F}_{m n}^{2}\right]\left(\lambda A^{4}\right)\left(\lambda A^{5}\right)\right\rangle \\
& +\left\langle\left[\left(\lambda A^{1}\right)\left(k^{1} \cdot A^{3}\right)\left(k^{3} \cdot A^{2}\right)+\frac{1}{2} k_{m}^{1}\left(\lambda A^{1}\right)\left(W^{2} \gamma^{m} W^{3}\right)\right]\left(\lambda A^{4}\right)\left(\lambda A^{5}\right)\right\rangle \\
& +\left\langle\left[\left(k^{2} \cdot k^{3}\right)\left(A^{1} W^{3}\right)\left(\lambda A^{2}\right)+\left(k^{1} \cdot k^{2}\right)\left(A^{2} W^{3}\right)\left(\lambda A^{1}\right)\right]\left(\lambda A^{4}\right)\left(\lambda A^{5}\right)\right\rangle-(2 \leftrightarrow 3)
\end{aligned}
$$

while the other $L_{i j k l}$ are obtained by exchanging labels appropriately. All the terms containing factors of $\left(k^{i} \cdot k^{j}\right)\left(A^{k} W^{l}\right)$ are "total derivative" terms and will be shown to cancel in the final result. Furthermore, the double pole in the OPE of $U^{2}\left(z_{2}\right) U^{3}\left(z_{3}\right)$ gives rise to the following expression for $L_{2314}$

$$
L_{2314}=\left(\alpha_{23}+1\right)\left\langle\left[\left(A^{2} W^{3}\right)+\left(A^{3} W^{2}\right)-\left(A^{2} \cdot A^{3}\right)\right]\left(\lambda A^{1}\right)\left(\lambda A^{4}\right)\left(\lambda A^{5}\right)\right\rangle \equiv\left(\alpha_{23}+1\right) L_{23} .
$$

As will become clear later, the factor of $\left(1+\alpha_{23}\right)$ appearing in $(2.4)$ is essential to obtain a simple answer for the amplitude. That this is possible can be traced back to the fact that the pure spinor Lorentz currents have level -3 (see the computations of appendix A).

With the notation of [17] for the integrals appearing ${ }^{3}$ in (2.2), the amplitude can be written as $\mathcal{A}_{5}=L_{2131} K_{1}-L_{2134} K_{2}-L_{2434} K_{1}^{\prime}+L_{2431} K_{3}-L_{2331} K_{5}-L_{2334} K_{4}^{\prime}+L_{2314} K_{6}$, or

$$
A_{5}=L_{2431} K_{3}-L_{2134} K_{2}+L_{2131} K_{4}-L_{3424} K_{4}^{\prime}+L_{2434} K_{5}^{\prime}-L_{3121} K_{5}+L_{2314} K_{6},
$$

where we used $K_{1}=K_{4}-K_{5}, K_{1}^{\prime}=K_{4}^{\prime}-K_{5}^{\prime}[18]$ and

$$
L_{2331}=L_{3121}-L_{2131}, \quad L_{2334}=L_{3424}-L_{2434},
$$

\footnotetext{
${ }^{3}$ The RNS computations of $[17,18]$ required the evaluation of more complicated integrals with cubic terms in the denominators.
} 
where (2.6) will be proved as an OPE identity in the next section. Plugging in the expressions for $K_{j}$ in terms of $T$ and $K_{3}$ derived in the appendix D, the amplitude (2.5) becomes

$$
\mathcal{A}_{5}(1,2,3,4,5)=T A_{\mathrm{YM}}(\theta)+K_{3} A_{F^{4}}(\theta),
$$

where $A_{\mathrm{YM}}(\theta)$ and $A_{F^{4}}(\theta)$ are superfields,

$$
\begin{aligned}
A_{\mathrm{YM}}(\theta)= & \frac{L_{2131}}{\alpha_{12} \alpha_{45}}-\frac{L_{3424}}{\alpha_{34} \alpha_{51}}-\frac{L_{2334}}{\alpha_{23} \alpha_{51}}-\frac{L_{2331}}{\alpha_{23} \alpha_{45}}-\frac{L_{2134}}{\alpha_{12} \alpha_{34}}-\frac{L_{23}}{\alpha_{23}}\left(\frac{\alpha_{24}}{\alpha_{51}}+\frac{\alpha_{13}}{\alpha_{45}}-1\right) \\
A_{F^{4}}(\theta)= & L_{2431}-L_{2331}-L_{2334}-L_{2134}+\frac{L_{23}}{\alpha_{23}}\left(\alpha_{13} \alpha_{24}-\alpha_{12} \alpha_{34}-\alpha_{23} \alpha_{34}-\alpha_{12} \alpha_{23}\right) \\
& +L_{2331}\left(\frac{\alpha_{12}}{\alpha_{45}}+\frac{\alpha_{51}}{\alpha_{23}}\right)+L_{2334}\left(\frac{\alpha_{34}}{\alpha_{51}}+\frac{\alpha_{45}}{\alpha_{23}}\right)+L_{2134}\left(\frac{\alpha_{45}}{\alpha_{12}}+\frac{\alpha_{51}}{\alpha_{34}}\right) \\
& +L_{3424}\left(\frac{\alpha_{12}}{\alpha_{34}}+\frac{\alpha_{23}}{\alpha_{51}}\right)-L_{2131}\left(\frac{\alpha_{34}}{\alpha_{12}}+\frac{\alpha_{23}}{\alpha_{45}}\right)+L_{23}\left(\frac{\alpha_{12} \alpha_{13}}{\alpha_{45}}+\frac{\alpha_{34} \alpha_{24}}{\alpha_{51}}\right) .
\end{aligned}
$$

From (2.8) and (2.9) all the other partial amplitudes can be obtained by permutation. Therefore (2.7) is the supersymmetric generalization of equation (4.13) of [18]. In the field theory limit the amplitude (2.7) reduces to $A_{\mathrm{YM}}(\theta)$.

Using the superspace expressions (2.3) and (2.4) one sees that all terms containing factors of $\left(k^{i} \cdot k^{j}\right)\left(A^{k} W^{l}\right)$ cancel out in (2.8) and (2.9). For example, the terms in (2.9) containing $\left(A^{3} W^{2}\right)$ are given by

$$
\left(\frac{\alpha_{34}}{\alpha_{51}}\left(\alpha_{23}+\alpha_{24}+\alpha_{34}\right)-\alpha_{34}\right)\left\langle\left(A^{3} W^{2}\right)\left(\lambda A^{1}\right)\left(\lambda A^{4}\right)\left(\lambda A^{5}\right)\right\rangle=0
$$

because $\alpha_{23}+\alpha_{24}+\alpha_{34}=\alpha_{51}$. In fact they come from total derivative terms, as can be seen in the explicit computations of appendix A.

The expressions (2.8) and (2.9) can be further simplified by absorbing the "contact terms" containing $L_{23}$ conveniently, taking the relations (2.6) as a guide. Using the identities $L_{3224}=-L_{2334}$ and $L_{3221}=-L_{2331}$, which follow trivially from the antisymmetry of the simple pole of $U^{2}\left(z_{2}\right) U^{3}\left(z_{3}\right)$ under $2 \leftrightarrow 3$, one can can rewrite $A_{\mathrm{YM}}(1,2,3,4,5)$ and $A_{\mathrm{YM}}(1,3,2,4,5)$ as

$$
\begin{aligned}
& A_{\mathrm{YM}}(1,2,3,4,5)=\frac{\tilde{L}_{2131}}{\alpha_{12} \alpha_{45}}-\frac{\tilde{L}_{3424}}{\alpha_{34} \alpha_{51}}-\frac{\tilde{L}_{2334}}{\alpha_{23} \alpha_{51}}-\frac{\tilde{L}_{2331}}{\alpha_{23} \alpha_{45}}-\frac{\tilde{L}_{2134}}{\alpha_{12} \alpha_{34}} \\
& A_{\mathrm{YM}}(1,3,2,4,5)=\frac{L_{3121}}{\alpha_{13} \alpha_{45}}-\frac{L_{2434}}{\alpha_{24} \alpha_{51}}+\frac{\tilde{L}_{2334}}{\alpha_{23} \alpha_{51}}+\frac{\tilde{L}_{2331}}{\alpha_{23} \alpha_{45}}-\frac{L_{3124}}{\alpha_{13} \alpha_{24}}
\end{aligned}
$$

where we used $\alpha_{24}=\alpha_{51}-\alpha_{23}-\alpha_{34}$ and $\alpha_{13}=\alpha_{45}-\alpha_{23}-\alpha_{12}$ and the redefined $\tilde{L}_{i j k l}$ are given by

$$
\begin{array}{ll}
\tilde{L}_{2131}=L_{2131}+\alpha_{12} L_{23}-\alpha_{45} L_{23}, & \tilde{L}_{3424}=L_{3424}-\alpha_{34} L_{23} \\
\tilde{L}_{2134}=L_{2134}-\alpha_{34} L_{23} & \\
\tilde{L}_{2334}=L_{2334}-\alpha_{34} L_{23}, & \tilde{L}_{2331}=L_{2331}-\alpha_{12} L_{23}+\alpha_{45} L_{23} .
\end{array}
$$


The identities (2.6) continue to hold with these redefinitions, that is

$$
\tilde{L}_{2331}=L_{3121}-\tilde{L}_{2131}, \quad \tilde{L}_{2334}=\tilde{L}_{3424}-L_{2434} .
$$

The use of (2.13) also removes the contact terms appearing in (2.9), simplifying it. In fact, using (2.13) the supersymmetric string theory partial amplitude (2.1) becomes,

$$
\begin{aligned}
\mathcal{A}_{5}(1,2,3,4,5)= & -\frac{\tilde{L}_{2134}}{\alpha_{12} \alpha_{34}}\left(T-K_{3}\left(\alpha_{34} \alpha_{45}+\alpha_{12}\left(\alpha_{51}+\alpha_{34}\right)\right)\right) \\
& +\frac{\tilde{L}_{2131}}{\alpha_{12} \alpha_{45}}\left(T-K_{3}\left(\alpha_{34} \alpha_{45}+\alpha_{23} \alpha_{12}\right)\right) \\
& -\frac{\tilde{L}_{3424}}{\alpha_{34} \alpha_{51}}\left(T-K_{3}\left(\alpha_{12} \alpha_{51}+\alpha_{23} \alpha_{34}\right)\right)+L_{2431} K_{3} \\
& -\frac{\tilde{L}_{2331}}{\alpha_{23} \alpha_{45}}\left(T-K_{3}\left(\alpha_{12} \alpha_{23}+\alpha_{45}\left(\alpha_{51}+\alpha_{23}\right)\right)\right) \\
& -\frac{\tilde{L}_{2334}}{\alpha_{23} \alpha_{51}}\left(T-K_{3}\left(\alpha_{12} \alpha_{23}+\alpha_{51}\left(\alpha_{45}+\alpha_{23}\right)\right)\right.
\end{aligned}
$$

The component expansions of (2.9) and (2.8) can be computed ${ }^{4}$ using the methods of $[3,29,30]$. When all external states are bosonic the RNS results of $[17,18]$ are recovered,

$$
\left.A_{\mathrm{YM}}(\theta)\right|_{\mathrm{NS}}=-\frac{1}{2880} A_{\mathrm{YM}}^{\mathrm{RNS}},\left.\quad A_{F^{4}}(\theta)\right|_{\mathrm{NS}}=-\frac{1}{2880} A_{F^{4}}^{\mathrm{RNS}} .
$$

The higher $\alpha^{\prime}$ expansion in (2.7) is determined solely by the expansions of $T$ and $K_{3}$, and all the (supersymmetric) information about the external states is encoded in the superfield expressions $A_{\mathrm{YM}}(\theta)$ and $A_{F^{4}}(\theta)$, in accord with the observations of [31]. This is in fact a generic feature of the amplitudes computed in the pure spinor formalism. The kinematic factors of bosonic and fermionic states are always multiplied by the same "form factors", which come from the integrals over the world-sheet.

\section{Derivation of the BCJ kinematic identities}

In reference [19], the massless four-point partial amplitudes at tree-level were represented in terms of its poles as

$$
A(1,2,3,4)=\frac{n_{s}}{s}+\frac{n_{t}}{t}, \quad A(1,3,4,2)=-\frac{n_{u}}{u}-\frac{n_{s}}{s}, \quad A(1,4,2,3)=-\frac{n_{t}}{t}+\frac{n_{u}}{u},
$$

and the identity $n_{u}=n_{s}-n_{t}$ was explicitly shown to be true. Furthermore, the five-point amplitudes were written as

$$
\begin{aligned}
& A_{\mathrm{YM}}(1,2,3,4,5)=\frac{n_{1}}{\alpha_{12} \alpha_{45}}+\frac{n_{2}}{\alpha_{23} \alpha_{51}}+\frac{n_{3}}{\alpha_{34} \alpha_{12}}+\frac{n_{4}}{\alpha_{23} \alpha_{45}}+\frac{n_{5}}{\alpha_{51} \alpha_{34}} \\
& A_{\mathrm{YM}}(1,3,2,4,5)=\frac{n_{15}}{\alpha_{13} \alpha_{45}}-\frac{n_{2}}{\alpha_{23} \alpha_{51}}-\frac{n_{10}}{\alpha_{24} \alpha_{13}}-\frac{n_{4}}{\alpha_{23} \alpha_{45}}-\frac{n_{11}}{\alpha_{51} \alpha_{24}} \\
& A_{\mathrm{YM}}(1,4,3,2,5)=\frac{n_{6}}{\alpha_{14} \alpha_{25}}+\frac{n_{5}}{\alpha_{34} \alpha_{51}}+\frac{n_{7}}{\alpha_{23} \alpha_{14}}+\frac{n_{8}}{\alpha_{25} \alpha_{34}}+\frac{n_{2}}{\alpha_{51} \alpha_{32}}
\end{aligned}
$$

\footnotetext{
${ }^{4}$ This task can be implemented in a computer program [26-28].
} 


$$
\begin{aligned}
& A_{\mathrm{YM}}(1,3,4,2,5)=\frac{n_{9}}{\alpha_{13} \alpha_{25}}-\frac{n_{5}}{\alpha_{34} \alpha_{51}}+\frac{n_{10}}{\alpha_{24} \alpha_{13}}-\frac{n_{8}}{\alpha_{25} \alpha_{34}}+\frac{n_{11}}{\alpha_{51} \alpha_{24}} \\
& A_{\mathrm{YM}}(1,2,4,3,5)=\frac{n_{12}}{\alpha_{12} \alpha_{35}}+\frac{n_{11}}{\alpha_{24} \alpha_{51}}-\frac{n_{3}}{\alpha_{34} \alpha_{12}}+\frac{n_{13}}{\alpha_{35} \alpha_{24}}-\frac{n_{5}}{\alpha_{51} \alpha_{34}} \\
& A_{\mathrm{YM}}(1,4,2,3,5)=\frac{n_{14}}{\alpha_{14} \alpha_{35}}-\frac{n_{11}}{\alpha_{24} \alpha_{51}}-\frac{n_{7}}{\alpha_{23} \alpha_{14}}-\frac{n_{13}}{\alpha_{35} \alpha_{24}}-\frac{n_{2}}{\alpha_{51} \alpha_{23}}
\end{aligned}
$$

and by analogy with the Jacobi-like four-point kinematic relation, the numerators were required to satisfy

$$
\begin{array}{rlrl}
n_{3}-n_{5}+n_{8} & =0, \\
n_{4}-n_{1}+n_{15} & =0, \\
n_{5}-n_{2}+n_{11} & =0, \\
n_{8}-n_{6}+n_{9} & =0, & n_{3}-n_{1}+n_{12} & =0, \\
n_{10}-n_{11}+n_{13} & =0, & n_{7}-n_{2}+n_{7}+n_{14} & =0, \\
n_{10}-n_{9}+n_{15} & =0, \\
n_{13}-n_{12}+n_{14} & =0,
\end{array}
$$

which was explicitly verified to be true. Extending the same reasoning to higher points, it was argued that those kind of relations impose additional constraints which reduce the number of independent $N$-point color-ordered amplitudes to $(N-3)$ !. This conclusion was later demonstrated in [20] using the field theory limit of string theory. We will now prove the identity (2.6) and discuss its relation with the 5-point BCJ identities of [19].

To prove (2.6) it suffices to note that in the computation of

$$
\left\langle V^{1}\left(z_{1}\right) V^{4}\left(z_{4}\right) V^{5}\left(z_{5}\right) U^{2}\left(z_{2}\right) U^{3}\left(z_{3}\right)\right\rangle,
$$

a kinematic identity can be obtained by considering the different orders in which the OPE's are evaluated. By computing first the OPE's of $U^{2}\left(z_{2}\right)$ followed by $U^{3}\left(z_{3}\right)$ one gets,

$$
\frac{L_{2131}}{z_{21} z_{31}}+\frac{L_{2134}}{z_{21} z_{34}}-\frac{L_{2434}}{z_{24} z_{34}}-\frac{L_{2431}}{z_{24} z_{31}}+\frac{L_{2331}}{z_{23} z_{31}}-\frac{L_{2334}}{z_{23} z_{34}}+\frac{L_{2314}}{z_{23}^{2}}
$$

while in reverse order,

$$
\frac{L_{3121}}{z_{31} z_{21}}+\frac{L_{3124}}{z_{31} z_{24}}-\frac{L_{3424}}{z_{34} z_{24}}-\frac{L_{3421}}{z_{34} z_{21}}+\frac{L_{3221}}{z_{32} z_{21}}-\frac{L_{3224}}{z_{32} z_{24}}+\frac{L_{3214}}{z_{32}^{2}} .
$$

As the integrated vertex $U^{I}$ is bosonic, (3.4) and (3.5) must be equal. Therefore we get

$$
\begin{aligned}
& \frac{\left(L_{2131}-L_{3121}\right)}{z_{21} z_{31}}-\frac{\left(L_{2434}-L_{3424}\right)}{z_{24} z_{34}} \\
& \quad+L_{2331}\left(\frac{1}{z_{23} z_{31}}+\frac{1}{z_{32} z_{21}}\right)-L_{2334}\left(\frac{1}{z_{23} z_{34}}+\frac{1}{z_{32} z_{24}}\right) \\
& \quad+\frac{1}{z_{34} z_{24}}\left(L_{2134}+L_{3421}\right)-\frac{1}{z_{24} z_{31}}\left(L_{2431}+L_{3124}\right)=0
\end{aligned}
$$

where we used $L_{3221}=-L_{2331}$ and $L_{2314}=L_{3214}$. To see this one notes that

$$
\left\langle\left[\left[U^{2}\left(z_{2}\right) U^{3}\left(z_{3}\right)\right] V^{1}\left(z_{1}\right) V^{4}\left(z_{4}\right) V^{5}\left(z_{5}\right)\right]\right\rangle=\left\langle\left[\left[U^{3}\left(z_{3}\right) U^{2}\left(z_{2}\right)\right] V^{1}\left(z_{1}\right) V^{4}\left(z_{4}\right) V^{5}\left(z_{5}\right)\right]\right\rangle
$$


implies

$$
\lim _{z_{2} \rightarrow z_{3}}\left[\frac{L_{2331}}{z_{23} z_{31}}+\frac{L_{2314}}{z_{23}^{2}}\right]=\lim _{z_{3} \rightarrow z_{2}}\left[\frac{L_{3221}}{z_{32} z_{21}}+\frac{L_{3214}}{z_{32}^{2}}\right]
$$

and therefore $L_{2331}=-L_{3221}$ and $L_{2314}=L_{3214}$. That is, the simple and double poles of the $U^{2}\left(z_{2}\right) U^{3}\left(z_{3}\right)$ OPE are antisymmetric and symmetric under $2 \leftrightarrow 3$, respectively. Finally, using $\frac{1}{z_{23} z_{31}}+\frac{1}{z_{32} z_{21}}=\frac{1}{z_{21} z_{31}}$ in (3.6) leads to

$$
\begin{aligned}
L_{2131}-L_{3121}+L_{2331} & =0, & L_{2434}-L_{3424}+L_{2334} & =0, \\
L_{2134} & =-L_{3421}, & L_{2431} & =-L_{3124} .
\end{aligned}
$$

The identities (3.7) and (3.8) can be also verified from their explicit pure spinor superspace expressions given in the appendix A.

After absorbing the contact terms as in (2.13), the field theory limit of the string partial amplitudes $A(1,2,3,4,5)$ and $A(1,3,2,4,5)$ are given by (2.11) and (2.12), respectively. Note that there is an ambiguity (or freedom) on how to absorb the contact terms, as there is no unique way in doing so. We chose to absorb them while preserving the kinematic identities $^{5}$ (3.7). This is in agreement with the discussions of [19], where it is emphasized that the BCJ identities would not be satisfied by any choice of absorbing contact terms.

Comparing (2.11) and (2.12) with (3.2) allow us to identify

$$
\begin{aligned}
& n_{1}=\tilde{L}_{2131}, \quad n_{4}=-\tilde{L}_{2331}, \quad n_{15}=L_{3121}, \\
& n_{5}=-\tilde{L}_{3424}, \quad n_{2}=-\tilde{L}_{2334}, \\
& n_{11}=L_{2434}=-L_{2443}, \quad n_{3}=-\tilde{L}_{2134}, \quad n_{10}=L_{3124}=-L_{2431} \text {. }
\end{aligned}
$$

where $L_{2443}=-L_{2434}$ follows from $\left\langle\left[\left[U^{2}\left(z_{2}\right) V^{4}\left(z_{4}\right)\right] U^{3}\left(z_{3}\right)\right]\right\rangle=\left\langle\left[U^{3}\left(z_{3}\right)\left[U^{2}\left(z_{2}\right) V^{4}\left(z_{4}\right)\right]\right\rangle\right.$. Therefore (2.14) is the supersymmetric generalization of the BCJ relations

$$
n_{4}-n_{1}+n_{15}=0, \quad n_{5}-n_{2}+n_{11}=0 .
$$

Using $U^{2}$ and $U^{4}$ (or $U^{3}$ and $U^{4}$ ) as integrated vertices whose positions run between 0 and 1 would lead to the BCJ identities $n_{14}+n_{13}-n_{12}=0$ and $n_{5}-n_{2}+n_{11}=0$ (or $n_{8}-n_{6}+n_{9}=0$ and $\left.n_{5}-n_{2}+n_{11}=0\right)$.

How can the remaining (supersymmetric) BCJ relations be obtained? The four-point derivation of the BCJ identity provides the hint, as there are no two integrated vertices to allow an OPE identity in this case. Using the results of [6] and the gamma function identity of $\Gamma(1+x)=x \Gamma(x)$ one can obtain the open string partial amplitudes from $\left\langle V^{1}(0) \int U^{2} V^{3}(1) V^{4}(\infty)\right\rangle$ by explicitly computing the integral over the three domains $0 \leq$ $z_{2} \leq 1,-\infty \leq z_{2} \leq 0$, and $1 \leq z_{2} \leq \infty$,

$$
\begin{aligned}
& A(1,2,3,4)=\left(-\frac{K_{21}}{s}+\frac{K_{23}}{t}\right) \frac{\Gamma(1-t) \Gamma(1-s)}{\Gamma(1+u)} \\
& A(1,3,4,2)=\left(-\frac{t K_{21}}{s u}+\frac{K_{23}}{u}\right) \frac{\Gamma(1-s) \Gamma(1-u)}{\Gamma(1+t)} \\
& A(1,3,2,4)=\left(-\frac{K_{21}}{u}+\frac{s K_{23}}{u t}\right) \frac{\Gamma(1-t) \Gamma(1-u)}{\Gamma(1+s)}
\end{aligned}
$$

\footnotetext{
${ }^{5}$ The full string theory computation provides one extra layer of motivation for the redefinitions of (2.13), as they also remove the contact terms from the stringy correction $A_{F^{4}}(\theta)$.
} 
where

$$
\begin{aligned}
& K_{21}=-\left\langle\left[A_{m}^{1}\left(\lambda \gamma^{m} W^{2}\right)+\left(\lambda A^{1}\right)\left(k^{1} \cdot A^{2}\right)\right]\left(\lambda A^{3}\right)\left(\lambda A^{4}\right)\right\rangle \\
& K_{23}=+\left\langle\left[A_{m}^{3}\left(\lambda \gamma^{m} W^{2}\right)+\left(\lambda A^{3}\right)\left(k^{3} \cdot A^{2}\right)\right]\left(\lambda A^{1}\right)\left(\lambda A^{4}\right)\right\rangle
\end{aligned}
$$

and $s=-2\left(k^{1} \cdot k^{2}\right)=-2\left(k^{3} \cdot k^{4}\right), u=-2\left(k^{1} \cdot k^{3}\right)=-2\left(k^{2} \cdot k^{4}\right), t=-2\left(k^{1} \cdot k^{4}\right)=-2\left(k^{2} \cdot k^{3}\right)$. Using $s+t+u=0$ and taking the field theory limit one can easily derive the supersymmetric generalization of the four-point BCJ relation $n_{u}=n_{s}-n_{t}$ by comparing (3.11) with (3.1). That is, $n_{s}=-K_{21}, n_{t}=K_{23}$ and $n_{u}=-K_{21}-K_{23}$.

Therefore, computing the integrals appearing in the five-point scattering amplitude for each of the twelve regions of integration should provide the remaining five-point BCJ identities in a supersymmetric fashion. For example, the partial amplitude $A_{\mathrm{YM}}(1,4,2,3,5)$ is obtained by integrating (2.2) over $1 \leq z_{2} \leq z_{3} \leq \infty$, and in this case the kinematic factors for the different poles appearing in the last equation of (3.2) will be given by combinations of the factors already present in (2.11), so that new identities will have to arise. In fact, using the transformations $y_{3}=\left(z_{3}-1\right) / z_{3}$ and $y_{2}=\left(z_{2}-1\right) / z_{2}$ the integrals

$$
\int_{1}^{\infty} d z_{3} \int_{1}^{z_{3}} d z_{2} z_{3}^{\alpha_{13}}\left(1-z_{3}\right)^{\alpha_{34}} z_{2}^{\alpha_{12}}\left(1-z_{2}\right)^{\alpha_{24}}\left(z_{3}-z_{2}\right)^{\alpha_{23}} F\left(z_{3}, z_{2}\right)
$$

become

$$
\int_{0}^{1} d y_{3} \int_{0}^{y_{3}} d y_{2} y_{3}^{\alpha_{34}}\left(1-y_{3}\right)^{\alpha_{35}} y_{2}^{\alpha_{24}}\left(1-y_{2}\right)^{\alpha_{25}}\left(y_{3}-y_{2}\right)^{\alpha_{23}} \frac{\tilde{F}\left(y_{3}, y_{2}\right)}{\left(1-y_{3}\right)^{2}\left(1-y_{2}\right)^{2}}
$$

which allow them to be written in terms of $K_{j}$ and $L_{j}$ of [17], provided that

$$
\alpha_{13} \rightarrow \alpha_{34}, \alpha_{34} \rightarrow \alpha_{35}, \alpha_{12} \rightarrow \alpha_{24}, \alpha_{24} \rightarrow \alpha_{25}, \alpha_{51} \rightarrow \alpha_{14}, \alpha_{23} \rightarrow \alpha_{23}, \alpha_{45} \rightarrow \alpha_{51}
$$

The only "new" integral which is not already computed in [17] is the one associated to $F\left(z_{3}, z_{2}\right)=\left(1-z_{2}\right)^{-1}\left(1-z_{3}\right)^{-1}$, namely

$$
\int_{0}^{1} d y_{3} \int_{0}^{y_{3}} d y_{2} y_{3}^{\alpha_{34}}\left(1-y_{3}\right)^{\alpha_{35}} y_{2}^{\alpha_{24}}\left(1-y_{2}\right)^{\alpha_{25}}\left(y_{3}-y_{2}\right)^{\alpha_{23}} \frac{1}{y_{2} y_{3}\left(1-y_{3}\right)\left(1-y_{2}\right)} .
$$

However, (3.13) is easily seen to be equal to $K_{1}^{\prime}+K_{3}+L_{3} \equiv L_{8}$. Finally, the amplitude (2.2) integrated over $1 \leq z_{2} \leq z_{3} \leq \infty$ is given by

$$
A(1,4,2,3,5)=L_{2131} \tilde{K}_{1}^{\prime}+L_{2134} \tilde{L}_{1}^{\prime}-L_{2434} \tilde{L}_{8}-L_{2431} \tilde{L}_{3}^{\prime}-L_{2331} \tilde{K}_{5}^{\prime}+L_{2334} \tilde{L}_{7}+L_{23}\left(1+\alpha_{23}\right) \tilde{K}_{6}
$$

where the tildes mean that the substitution (3.12) must be performed. Using the explicit results of [17] for the integrals, the field theory limit of (3.14) is given by

$$
\begin{aligned}
A_{\mathrm{YM}}(1,4,2,3,5)= & \frac{\left(L_{2131}+L_{2134}-L_{2434}-L_{2431}\right)}{\alpha_{14} \alpha_{35}}-\frac{L_{2434}}{\alpha_{24} \alpha_{51}}+\frac{\left(L_{2334}-L_{2331}\right)}{\alpha_{14} \alpha_{23}} \\
& -\frac{\left(L_{2434}+L_{2431}\right)}{\alpha_{24} \alpha_{35}}+\frac{L_{2334}}{\alpha_{23} \alpha_{51}}+L_{23}\left(\frac{1}{\alpha_{14}}+\frac{\alpha_{35}}{\alpha_{14} \alpha_{23}}-\frac{\alpha_{34}}{\alpha_{23} \alpha_{51}}\right) .
\end{aligned}
$$


With the redefinitions of (2.13), the contact terms are completely absorbed and (3.15) becomes

$$
\begin{aligned}
& A_{\mathrm{YM}}(1,4,2,3,5)= \\
& =\frac{\left(\tilde{L}_{2131}+\tilde{L}_{2134}-L_{2434}-L_{2431}\right)}{\alpha_{14} \alpha_{35}}-\frac{L_{2434}}{\alpha_{24} \alpha_{51}}+\frac{\left(\tilde{L}_{2334}-\tilde{L}_{2331}\right)}{\alpha_{14} \alpha_{23}} \\
& -\frac{\left(L_{2434}+L_{2431}\right)}{\alpha_{24} \alpha_{35}}+\frac{\tilde{L}_{2334}}{\alpha_{23} \alpha_{51}} .
\end{aligned}
$$

From exchanging $2 \leftrightarrow 3$ in (3.15) and using (2.13) it follows that

$$
\begin{aligned}
& A_{\mathrm{YM}}(1,4,3,2,5)= \\
& =\frac{\left(L_{3121}+L_{3124}-\tilde{L}_{3424}+\tilde{L}_{2134}\right)}{\alpha_{14} \alpha_{25}}-\frac{\tilde{L}_{3424}}{\alpha_{34} \alpha_{51}}-\frac{\left(\tilde{L}_{2334}-\tilde{L}_{2331}\right)}{\alpha_{14} \alpha_{23}} \\
& -\frac{\left(\tilde{L}_{3424}-\tilde{L}_{2134}\right)}{\alpha_{34} \alpha_{25}}-\frac{\tilde{L}_{2334}}{\alpha_{23} \alpha_{51}}
\end{aligned}
$$

Finally, comparing the above with (3.2) results in the new identifications

$$
\begin{aligned}
n_{7} & =\tilde{L}_{2331}-\tilde{L}_{2334}, & & n_{13}=L_{2434}+L_{2431}, \\
n_{14} & =\tilde{L}_{2131}+\tilde{L}_{2134}-L_{2434}-L_{2431}, & & \\
n_{6} & =L_{3121}+L_{3124}-\tilde{L}_{3424}+\tilde{L}_{2134}, & & n_{8}=-L_{3424}+\tilde{L}_{2134}
\end{aligned}
$$

and therefore the following BCJ identities are obtained

$$
\begin{array}{rlrl}
n_{6} & =n_{15}+n_{10}+n_{5}-n_{3}, \\
n_{7} & =-n_{4}+n_{2}, & n_{8} & =-n_{3}+n_{5} \\
n_{13} & =n_{11}-n_{10}, \quad n_{14}=n_{1}-n_{3}-n_{11}+n_{10} .
\end{array}
$$

Solving (3.17) and (3.10) in terms of $n_{1}, \ldots, n_{6}$ gives

$$
\begin{aligned}
& n_{7}=n_{2}-n_{4}, \quad n_{8}=-n_{3}+n_{5}, \\
& n_{10}=-n_{1}+n_{3}+n_{4}-n_{5}+n_{6}, \quad n_{11}=n_{2}-n_{5}, \\
& n_{13}=n_{1}+n_{2}-n_{3}-n_{4}-n_{6}, \quad n_{14}=-n_{2}+n_{4}+n_{6}, \quad n_{14}=n_{1}-n_{4}
\end{aligned}
$$

and together with $n_{8}-n_{6}+n_{9}=0$ and $n_{14}+n_{13}-n_{12}=0$, which follow as OPE identities using $U^{2}$ and $U^{4}$ or $U^{3}$ and $U^{4}$ as integrated vertices, we get the same solution as (4.12) of [19]. Therefore the BCJ identities of [19] were obtained from first principles. And by using the pure spinor formalism and its pure spinor superspace, we have shown that the BCJ relations are in fact supersymmetric.

\section{Acknowledgments}

I deeply thank Pierre Vanhove for reading an early draft, for suggesting the connection between the identities (2.6) and the BCJ relations and for pointing out the relevance of the different integration regions to obtain other BJC identities. I thank the organizers of the workshop Hidden Structures in Field Theory Amplitudes 2009, where I presented parts of this work. I also thank John Carrasco and Henrik Johansson for conversations during the workshop and Stefan Theisen and Nathan Berkovits for reading the draft. I acknowledge support by the Deutsch-Israelische Projektkooperation (DIP H52). 


\section{A Computation of the kinematic factors}

In this section we compute the OPE's appearing in the amplitude (2.1) to obtain the explicit expression for the kinematic factors $L_{i j k l}$ in pure spinor superspace.

Using the OPE's

$$
\begin{aligned}
& d_{\alpha}(z) V(w) \rightarrow \frac{D_{\alpha} V(w)}{z-w}, \quad \Pi^{m}(z) V(w) \rightarrow-\frac{k^{m} V(w)}{z-w}, \quad d_{\alpha}(z) \Pi^{m}(w) \rightarrow \frac{\left(\gamma^{m} \partial \theta\right)_{\alpha}}{z-w} \\
& d_{\alpha}(z) d_{\beta}(w) \rightarrow-\frac{\gamma_{\alpha \beta}^{m} \Pi_{m}}{z-w}, \quad \Pi^{m}(z) \Pi^{n}(w) \rightarrow-\frac{\eta^{m n}}{(z-w)^{2}}, \quad d_{\alpha}(z) \theta^{\beta}(w) \rightarrow \frac{\delta_{\alpha}^{\beta}}{(z-w)} \\
& d_{\alpha}(z) \partial \theta^{\beta}(w) \rightarrow \frac{\delta_{\alpha}^{\beta}}{(z-w)^{2}}, \quad w(z)_{\alpha} \lambda^{\beta}(w) \rightarrow-\frac{\delta_{\alpha}^{\beta}}{z-w}, \quad N^{m n}(z) \lambda^{\alpha}(w) \rightarrow-\frac{1}{2} \frac{\left(\lambda \gamma^{m n}\right)^{\alpha}}{z-w} \\
& N^{m n}(z) N_{p q}(w) \rightarrow+\frac{4}{z-w} N_{[p}^{[m} \delta_{q]}^{n]}-\frac{6}{(z-w)^{2}} \delta_{[p}^{n} \delta_{q]}^{m}
\end{aligned}
$$

and the equations of motion

$$
\begin{aligned}
D_{\alpha} A_{\beta}+D_{\beta} A_{\alpha} & =\gamma_{\alpha \beta}^{m} A_{m}, & D_{\alpha} A_{m} & =\left(\gamma_{m} W\right)_{\alpha}+k_{m} A_{\alpha}, \\
D_{\alpha} \mathcal{F}_{m n} & =2 k_{[m}\left(\gamma_{n]} W\right)_{\alpha}, & D_{\alpha} W^{\beta} & =\frac{1}{4}\left(\gamma^{m n}\right)_{\alpha}{ }^{\beta} \mathcal{F}_{m n},
\end{aligned}
$$

a long computation leads to the OPE between two integrated vertices,

$$
\begin{aligned}
U^{2}(z) U^{3}(w) \rightarrow \frac{1}{(z-w)}[ & \left(k^{2} \cdot A^{3}\right) U^{2}-\left(k^{3} \cdot A^{2}\right) U^{3}-\left(W^{2} \gamma_{m} W^{3}\right) \Pi^{m} \\
& -\partial \theta^{\alpha} D_{\alpha} A_{\beta}^{2} W_{3}^{\beta}-\Pi^{m} k_{m}^{2} A_{\alpha}^{2} W_{3}^{\alpha}+\partial \theta^{\alpha} D_{\alpha} A_{\beta}^{3} W_{2}^{\beta}+\Pi^{m} k_{m}^{3} A_{\alpha}^{3} W_{2}^{\alpha} \\
& +\frac{1}{4}\left(d \gamma^{m n} W^{2}\right) \mathcal{F}_{m n}^{3}-\frac{1}{4}\left(d \gamma^{m n} W^{3}\right) \mathcal{F}_{m n}^{2} \\
& \left.-\left(k_{m}^{2}+k_{m}^{3}\right)\left(W^{2} \gamma_{n} W^{3}\right) N^{m n}+\mathcal{F}_{m a}^{2} \mathcal{F}_{n a}^{3} N^{m n}\right] \\
+\frac{1}{(z-w)^{2}} & \left(1+\left(k^{2} \cdot k^{3}\right)\right)\left[\left(A^{2} W^{3}\right)+\left(A^{3} W^{2}\right)-\left(A^{2} \cdot A^{3}\right)\right] .
\end{aligned}
$$

where we dropped the total derivative terms with respect to $z_{2}$ which appear when Taylor expanding the superfields in the double pole. The super-Yang-Mills equations of motion (A.1) have been used judiciously to arrive at the simple answer (A.2). For example, the terms which contribute to the double pole are given by,

$$
\begin{gathered}
-A_{\alpha}^{2}\left[\partial \theta^{\alpha}(z) d_{\beta}(w)\right] W_{3}^{\beta}-W_{2}^{\alpha}\left[d_{\alpha}(z) \partial \theta^{\beta}(w)\right] A_{\beta}^{3}+\left[\Pi^{m}(z) \Pi^{n}(w)\right] A_{m}^{2} A_{n}^{3} \\
+\left[d_{\beta}(w) A_{m}^{2}(z)\right]\left[\Pi^{m}(z) W_{3}^{\beta}(w)\right]-\left[\Pi^{n}(w) W_{2}^{\alpha}(z)\right]\left[d_{\alpha}(z) A_{n}^{3}(w)\right] \\
+\left[\Pi^{m}(z) A_{n}^{3}(w)\right]\left[\Pi^{n}(w) A_{m}^{2}(z)\right]-\left[d_{\beta}(w) W_{2}^{\alpha}(z)\right]\left[d_{\alpha}(z) W_{3}^{\beta}(w)\right] \\
+\frac{1}{4}\left[N^{m n}(z) N^{p q}(w)\right] \mathcal{F}_{m n}^{2} \mathcal{F}_{p q}^{3}
\end{gathered}
$$

Using the OPE's one obtains (omitting $\left.(z-w)^{-2}\right)$

$$
\begin{aligned}
\left(A^{2} W^{3}\right)+\left(A^{3} W^{2}\right)- & \left(A^{2} \cdot A^{3}\right)+k_{m}^{3}\left(D_{\beta} A_{m}^{2}\right) W_{3}^{\beta}+k_{m}^{2}\left(D_{\alpha} A_{m}^{3}\right) W_{2}^{\alpha} \\
& -\left(k^{3} \cdot A^{2}\right)\left(k^{2} \cdot A^{3}\right)+\frac{1}{16} \operatorname{tr}\left(\gamma^{m n} \gamma^{p q}\right) \mathcal{F}_{m n}^{2} \mathcal{F}_{p q}^{3}+\frac{3}{2} \mathcal{F}_{m n}^{2} \mathcal{F}_{3}^{m n}
\end{aligned}
$$


where the last term comes from the level -3 double pole of the pure spinor Lorentz currents. One can now use $D_{\alpha} A_{m}=\left(\gamma_{m} W\right)_{\alpha}+k_{m} A_{\alpha}$ and the fact that $k_{m}\left(\gamma^{m} W\right)_{\alpha}=0$ to simplify (A.3) to,

$$
\begin{gathered}
\left(1+\left(k^{2} \cdot k^{3}\right)\right)\left[\left(A^{2} W^{3}\right)+\left(A^{3} W^{2}\right)\right]-\left(A^{2} \cdot A^{3}\right)-\left(k^{3} \cdot A^{2}\right)\left(k^{2} \cdot A^{3}\right)-\frac{1}{2} \mathcal{F}_{m n}^{2} \mathcal{F}_{3}^{m n} \\
=\left(1+\left(k^{2} \cdot k^{3}\right)\right)\left[\left(A^{2} W^{3}\right)+\left(A^{3} W^{2}\right)-\left(A^{2} \cdot A^{3}\right)\right]
\end{gathered}
$$

where we used $\operatorname{tr}\left(\gamma^{m n} \gamma_{p q}\right)=-32 \delta_{p q}^{m n}$ and $-\frac{1}{2}\left(F^{2} \cdot F^{3}\right)=-\left(k^{2} \cdot k^{3}\right)\left(A^{2} \cdot A^{3}\right)+\left(k^{2} \cdot A^{3}\right)\left(k^{3} \cdot A^{2}\right)$.

Using the same kind of manipulations as [6] one can also prove the following OPE identity as $z_{2} \rightarrow z_{1}$

$$
\left\langle\left(\lambda A^{1}\right)\left(z_{1}\right) U^{2}\left(z_{2}\right) \mathcal{M}\right\rangle=-\frac{1}{z_{21}}\left\langle\left[A_{m}^{1}\left(\lambda \gamma^{m} W^{2}\right)+\left(\lambda A^{1}\right)\left(k^{1} \cdot A^{2}\right)\right]\left(z_{1}\right) \mathcal{M}+\left(A^{1} W^{2}\right)\left(z_{1}\right) Q \mathcal{M}\right\rangle
$$

where $\mathcal{M}(x, \theta)$ is any superfield. Furthermore, if $Q \mathcal{M}=0$ then the following holds true

$$
\begin{aligned}
\left\langle\left(\lambda A^{1}\right)\left(z_{1}\right)[\right. & \left.\left.\frac{1}{4}\left(d \gamma^{m n} W^{2}\right) \mathcal{F}_{m n}^{3}-\frac{1}{4}\left(d \gamma^{m n} W^{3}\right) \mathcal{F}_{m n}^{2}+\mathcal{F}_{m a}^{2} \mathcal{F}_{n a}^{3} N^{m n}\right]\left(z_{3}\right) \mathcal{M}\right\rangle \\
& =+\left\langle\frac{1}{4}\left(\lambda \gamma^{p} \gamma^{m n} W^{3}\right) A_{p}^{1} \mathcal{F}_{m n}^{2} \mathcal{M}+\frac{1}{2} k_{m}^{2}\left(A^{1} \gamma^{m n} W^{3}\right)\left(\lambda \gamma_{n} W^{2}\right) \mathcal{M}\right\rangle-(2 \leftrightarrow 3)
\end{aligned}
$$

Also,

$$
-\left\langle\left(\lambda A^{1}\right)\left(k_{m}^{2}+k_{m}^{3}\right)\left(W^{2} \gamma_{n} W^{3}\right) N^{m n} M\right\rangle=\frac{1}{2\left(z_{3}-z_{1}\right)}\left\langle\left(\lambda \gamma^{m n} A^{1}\right)\left(k_{m}^{2}+k_{m}^{3}\right)\left(W^{2} \gamma_{n} W^{3}\right) M\right\rangle
$$

and

$$
\begin{aligned}
\left\langle\left(\lambda A^{1}\right)\left(z_{1}\right)\left(k^{2} \cdot A^{3}\right) U^{2}\left(z_{3}\right)\right\rangle= & -\frac{1}{z_{3}-z_{1}}\left\langle\left(A_{m}^{1}\left(\lambda \gamma^{m} W^{2}\right)+\left(\lambda A^{1}\right)\left(k^{1} \cdot A^{2}\right)\right)\left(k^{2} \cdot A^{3}\right)\right\rangle \\
& -\frac{1}{z_{3}-z_{1}}\left\langle\left(A^{1} W^{2}\right)\left(\left(k^{2} \cdot k^{3}\right)\left(\lambda A^{3}\right)+k_{m}^{2}\left(\lambda \gamma^{m} W^{3}\right)\right)\right\rangle .
\end{aligned}
$$

One can also show by using gamma matrix identities, the pure spinor constraint and the SYM equations of motion (A.1) that

$$
\begin{aligned}
& +\frac{1}{2} k_{m}^{2}\left(A^{1} \gamma^{m n} W^{3}\right)\left(\lambda \gamma^{n} W^{2}\right)-\frac{1}{2} k_{m}^{3}\left(A^{1} \gamma^{m n} W^{2}\right)\left(\lambda \gamma^{n} W^{3}\right) \\
& +\frac{1}{2}\left(\lambda \gamma^{m n} A^{1}\right)\left(k_{m}^{2}+k_{m}^{3}\right)\left(W^{2} \gamma_{n} W^{3}\right)+\left(A^{1} W^{3}\right) k_{m}^{3}\left(\lambda \gamma^{m} W^{2}\right)-\left(A^{1} W^{2}\right) k_{m}^{2}\left(\lambda \gamma^{m} W^{3}\right)=0 .
\end{aligned}
$$


From (A.6), (A.7), (A.8), (A.9) and the expression for the double pole (A.4) we finally get

$$
\begin{aligned}
\left\langle\left(\lambda A^{1}\right)\right. & \left.\left(\lambda A^{4}\right)\left(\lambda A^{5}\right)\left[U^{2} U^{3}\right]\left(z_{3}\right)\right\rangle= \\
& +\frac{1}{z_{23} z_{31}}\left[\left\langle\left[\frac{1}{4}\left(\lambda \gamma^{p} \gamma^{m n} W^{3}\right) A_{p}^{1} \mathcal{F}_{m n}^{2}-\frac{1}{4}\left(\lambda \gamma^{p} \gamma^{m n} W^{2}\right) A_{p}^{1} \mathcal{F}_{m n}^{3}\right]\left(\lambda A^{4}\right)\left(\lambda A^{5}\right)\right\rangle\right. \\
& +\left\langle\left(A_{m}^{1}\left(\lambda \gamma^{m} W^{2}\right)+\left(\lambda A^{1}\right)\left(k^{1} \cdot A^{2}\right)\right)\left(k^{2} \cdot A^{3}\right)\left(\lambda A^{4}\right)\left(\lambda A^{5}\right)\right\rangle \\
& -\left\langle\left(A_{m}^{1}\left(\lambda \gamma^{m} W^{3}\right)+\left(\lambda A^{1}\right)\left(k^{1} \cdot A^{3}\right)\right)\left(k^{3} \cdot A^{2}\right)\left(\lambda A^{4}\right)\left(\lambda A^{5}\right)\right\rangle \\
& \left.+\left\langle\left[k_{m}^{1}\left(\lambda A^{1}\right)\left(W^{2} \gamma^{m} W^{3}\right)+\left(k^{2} \cdot k^{3}\right)\left(A^{1} W^{3}\right)-\left(k^{2} \cdot k^{3}\right)\left(A^{1} W^{2}\right)\right]\left(\lambda A^{4}\right)\left(\lambda A^{5}\right)\right\rangle\right] \\
& +\frac{1}{z_{23}^{2}}\left\langle\left(\lambda A^{1}\right)\left(\lambda A^{4}\right)\left(\lambda A^{5}\right)\left[\left(A^{2} W^{3}\right)+\left(A^{3} W^{2}\right)-\left(A^{2} \cdot A^{3}\right)\right]\left(1+\left(k^{2} \cdot k^{3}\right)\right)\right\rangle-(1 \leftrightarrow 4)
\end{aligned}
$$

from which the following expressions can be read for $L_{2331}$ and $L_{2314}$,

$$
\begin{aligned}
L_{2331}= & A_{m}^{1} \mathcal{F}_{m n}^{2}\left(\lambda \gamma^{n} W^{3}\right)\left(\lambda A^{4}\right)\left(\lambda A^{5}\right)-\frac{1}{2}\left(\lambda \gamma_{m} W^{1}\right)\left(W^{2} \gamma^{m} W^{3}\right)\left(\lambda A^{4}\right)\left(\lambda A^{5}\right) \\
& \left.+\left[A_{m}^{1}\left(\lambda \gamma^{m} W^{3}\right)+\left(\lambda A^{1}\right)\left(k^{1} \cdot A^{3}\right)\right]\left(k^{3} \cdot A^{2}\right)\left(\lambda A^{4}\right)\left(\lambda A^{5}\right)\right\rangle \\
& \left.\left.+\left(k^{2} \cdot k^{3}\right)\left(A^{1} W^{3}\right)\left(\lambda A^{2}\right)\left(\lambda A^{4}\right)\left(\lambda A^{5}\right)\right\rangle+\left(k^{1} \cdot k^{2}\right)\left(A^{2} W^{3}\right)\left(\lambda A^{1}\right)\left(\lambda A^{4}\right)\left(\lambda A^{5}\right)\right\rangle \\
& -(2 \leftrightarrow 3)
\end{aligned}
$$

and

$$
L_{2314}=\left(1+\left(k^{2} \cdot k^{3}\right)\right)\left\langle\left(\lambda A^{1}\right)\left(\lambda A^{4}\right)\left(\lambda A^{5}\right)\left[\left(A^{2} W^{3}\right)+\left(A^{3} W^{2}\right)-\left(A^{2} \cdot A^{3}\right)\right]\right\rangle
$$

where we used that

$$
\begin{gathered}
\left.\left[\frac{1}{4}\left(\lambda \gamma^{p} \gamma^{m n} W^{3}\right) A_{p}^{1} \mathcal{F}_{m n}^{2}-\frac{1}{4}\left(\lambda \gamma^{p} \gamma^{m n} W^{2}\right) A_{p}^{1} \mathcal{F}_{m n}^{3}+k_{m}^{1}\left(\lambda A^{1}\right)\left(W^{2} \gamma^{m} W^{3}\right)\right]\left(\lambda A^{4}\right)\left(\lambda A^{5}\right)\right\rangle \\
=A_{m}^{1} \mathcal{F}_{m n}^{2}\left(\lambda \gamma^{n} W^{3}\right)\left(\lambda A^{4}\right)\left(\lambda A^{5}\right)-\frac{1}{2}\left(\lambda \gamma_{m} W^{1}\right)\left(W^{2} \gamma^{m} W^{3}\right)\left(\lambda A^{4}\right)\left(\lambda A^{5}\right)-(2 \leftrightarrow 3),
\end{gathered}
$$

which can be checked by writing $k_{m}^{1}\left(\lambda A^{1}\right)=Q A_{m}^{1}-\left(\lambda \gamma^{m} W^{1}\right)$ in the last term of the 1.h.s. and integrating the BRST charge by parts.

The expression for $L_{2131}$ can be deduced from the OPE as $z_{2} \rightarrow z_{1}$ followed by $z_{3} \rightarrow z_{1}$. Using (A.5) we obtain the singularity as $z_{2} \rightarrow z_{1}$

$$
\begin{aligned}
-\frac{1}{z_{21}}\left\langle\left[A_{m}^{1}\left(\lambda \gamma^{m} W^{2}\right)+\left(k^{1} \cdot A^{2}\right)\left(\lambda A^{1}\right)\right]\left(z_{1}\right) U^{3}\left(z_{3}\right)\left(\lambda A^{4}\right)\left(\lambda A^{5}\right)\right\rangle & \\
- & \frac{1}{z_{21}}\left\langle\left(A^{1} W^{2}\right)\left(z_{1}\right) \partial\left(\lambda A^{3}\right)\left(z_{3}\right)\left(\lambda A^{4}\right)\left(\lambda A^{5}\right)\right\rangle
\end{aligned}
$$

whose OPE computation for $z_{3} \rightarrow z_{1}$ implies, after some manipulations in superspace, that

$$
\begin{aligned}
L_{2131}=[ & \left.A_{m}^{1}\left(\lambda \gamma^{m} W^{2}\right)+\left(\lambda A^{1}\right)\left(k^{1} \cdot A^{2}\right)\right]\left(\lambda A^{4}\right)\left(\lambda A^{5}\right)\left(\left(k^{1}+k^{2}\right) \cdot A^{3}\right) \\
& -\left(W^{1} \gamma^{m} W^{2}\right)\left(\lambda \gamma_{m} W^{3}\right)\left(\lambda A^{4}\right)\left(\lambda A^{5}\right)+\left(A^{1} \cdot A^{2}\right) k_{m}^{2}\left(\lambda \gamma^{m} W^{3}\right)\left(\lambda A^{4}\right)\left(\lambda A^{5}\right) \\
& +A_{m}^{1}\left(\lambda \gamma^{m} W^{3}\right)\left(k^{1} \cdot A^{2}\right)\left(\lambda A^{4}\right)\left(\lambda A^{5}\right)-A_{m}^{2}\left(\lambda \gamma^{m} W^{3}\right)\left(k^{2} \cdot A^{1}\right)\left(\lambda A^{4}\right)\left(\lambda A^{5}\right) \\
& +\left(k^{1} \cdot k^{2}\right)\left(A^{1} W^{3}\right)\left(\lambda A^{2}\right)\left(\lambda A^{4}\right)\left(\lambda A^{5}\right)-\left(k^{1} \cdot k^{2}\right)\left(A^{2} W^{3}\right)\left(\lambda A^{1}\right)\left(\lambda A^{4}\right)\left(\lambda A^{5}\right) \\
& +\left(k^{1} \cdot k^{3}\right)\left(A^{1} W^{2}\right)\left(\lambda A^{3}\right)\left(\lambda A^{4}\right)\left(\lambda A^{5}\right)+\left(k^{2} \cdot k^{3}\right)\left(A^{1} W^{2}\right)\left(\lambda A^{3}\right)\left(\lambda A^{4}\right)\left(\lambda A^{5}\right),
\end{aligned}
$$


while $L_{2434}$ and $L_{3121}$ are obtained by exchanging $1 \leftrightarrow 4$ and $2 \leftrightarrow 3$, respectively.

The kinematic factor $L_{2134}$ is given by the coefficient of the OPE

$$
\left\langle\left(\lambda A^{1}\right)\left(z_{1}\right)\left(\lambda A^{4}\right)\left(z_{4}\right)\left(\lambda A^{5}\right)\left(z_{5}\right) U^{2}\left(z_{2}\right) U^{3}\left(z_{3}\right)\right\rangle
$$

as $z_{2} \rightarrow z_{1}$ followed by $z_{3} \rightarrow z_{4}$. Using (A.5) the first limit becomes

$$
\begin{aligned}
-\frac{1}{z_{21}}\left\langle\left[ A_{m}^{1}\left(\lambda \gamma^{m} W^{2}\right)+\left(\lambda A^{1}\right)\right.\right. & \left.\left.\left(k^{1} \cdot A^{2}\right)\right]\left(z_{1}\right)\left(\lambda A^{4}\right)\left(z_{4}\right)\left(\lambda A^{5}\right)\left(z_{5}\right) U^{3}\left(z_{3}\right)\right\rangle \\
& -\frac{1}{z_{21}}\left\langle\left(A^{1} W^{2}\right)\left(z_{1}\right)\left(\lambda A^{4}\right)\left(z_{4}\right)\left(\lambda A^{5}\right)\left(z_{5}\right) \partial\left(\lambda A^{3}\right)\left(z_{3}\right)\right\rangle .
\end{aligned}
$$

and using (A.5) again to evaluate as $z_{3} \rightarrow z_{4}$ we obtain

$$
\begin{aligned}
& +\frac{1}{z_{21} z_{34}}\left\langle\left[A_{m}^{1}\left(\lambda \gamma^{m} W^{2}\right)+\left(\lambda A^{1}\right)\left(k^{1} \cdot A^{2}\right)\right]\left[A_{m}^{4}\left(\lambda \gamma^{m} W^{3}\right)+\left(\lambda A^{4}\right)\left(k^{4} \cdot A^{3}\right)\right]\left(\lambda A^{5}\right)\right\rangle \\
& +\frac{1}{z_{21} z_{34}}\left[\left(k^{1} \cdot k^{2}\right)\left\langle\left(A^{4} W^{3}\right)\left(\lambda A^{1}\right)\left(\lambda A^{2}\right)\left(\lambda A^{5}\right)\right\rangle+\left(k^{3} \cdot k^{4}\right)\left\langle\left(A^{1} W^{2}\right)\left(\lambda A^{3}\right)\left(\lambda A^{4}\right)\left(\lambda A^{5}\right)\right\rangle\right],
\end{aligned}
$$

where we used $Q U^{3}=\partial\left(\lambda A^{3}\right)=\left(\partial \lambda^{\alpha}\right) A_{\alpha}^{3}+\Pi^{m} k_{m}^{3}\left(\lambda A^{3}\right)+\partial \theta^{\alpha} D_{\alpha}\left(\lambda A^{3}\right)$ and that $Q\left[A_{m}^{1}\left(\lambda \gamma^{m} W^{2}\right)+\left(\lambda A^{1}\right)\left(k^{1} \cdot A^{2}\right)\right]=-\left(k^{1} \cdot k^{2}\right)\left(\lambda A^{1}\right)\left(\lambda A^{2}\right)$. From (A.13) we get the expression for $L_{2134}$,

$$
\begin{aligned}
L_{2134}= & \left\langle\left[A_{m}^{1}\left(\lambda \gamma^{m} W^{2}\right)+\left(\lambda A^{1}\right)\left(k^{1} \cdot A^{2}\right)\right]\left[A_{m}^{4}\left(\lambda \gamma^{m} W^{3}\right)+\left(\lambda A^{4}\right)\left(k^{4} \cdot A^{3}\right)\right]\left(\lambda A^{5}\right)\right\rangle \\
& +\left(k^{1} \cdot k^{2}\right)\left\langle\left(A^{4} W^{3}\right)\left(\lambda A^{1}\right)\left(\lambda A^{2}\right)\left(\lambda A^{5}\right)\right\rangle+\left(k^{3} \cdot k^{4}\right)\left\langle\left(A^{1} W^{2}\right)\left(\lambda A^{3}\right)\left(\lambda A^{4}\right)\left(\lambda A^{5}\right)\right\rangle
\end{aligned}
$$

\section{A.1 Explicit proof of $L_{2331}=L_{3121}-L_{2131}$}

From the expressions (A.10) and (A.12) ( $L_{3121}$ is obtained from $L_{2131}$ by exchanging $(2 \leftrightarrow$ 3)) one can immediately check the following pure spinor superspace identity

$$
L_{2331}=L_{3121}-L_{2131} .
$$

To see this first note that all terms containing $\left(k^{i} \cdot k^{j}\right)$ trivially match on both sides of (A.14). Using that $\left(\lambda \gamma^{m} W^{2}\right)\left(W^{3} \gamma_{m} W^{1}\right)+\left(\lambda \gamma^{m} W^{3}\right)\left(W^{1} \gamma_{m} W^{2}\right)=\left(\lambda \gamma^{m} W^{1}\right)\left(W^{2} \gamma_{m} W^{3}\right)$ we get, after some trivial cancellations,

$$
\begin{aligned}
L_{3121}-L_{2131}-L_{2331}= & -\left(\lambda A^{1}\right)\left(\lambda A^{4}\right)\left(\lambda A^{5}\right)\left(k^{1} \cdot A^{2}\right)\left(k^{1} \cdot A^{3}\right) \\
& +\left[A_{m}^{2}\left(\lambda \gamma^{m} W^{3}\right)\left(k^{2} \cdot A^{1}\right)-\left(A^{1} \cdot A^{2}\right) k_{m}^{2}\left(\lambda \gamma^{m} W^{3}\right)\right. \\
& \left.-A_{m}^{1}\left(\lambda \gamma^{n} W^{3}\right) \mathcal{F}_{m n}^{2}\right]\left(\lambda A^{4}\right)\left(\lambda A^{5}\right)-(2 \leftrightarrow 3)
\end{aligned}
$$

which after using $\mathcal{F}_{m n}^{2}=k_{m}^{2} A_{n}^{2}-k_{n}^{2} A_{m}^{2}$ is equal to zero, as we wanted to show.

\section{B A different pure spinor superspace expression for $A_{F^{4}}$}

A different superfield expression for (2.9) may be suggested using the following argument. The one-loop amplitude of five massless states must factorize correctly in the massless poles, which appear when the surface degenerates into a one-loop four-point amplitude connected 
to a three-point at tree-level. This same factorization of the five-point one-loop amplitude probes the non-linear expansion (with five fields) of the one-loop interaction $F^{4}$. But the kinematic factors of four-point amplitudes at one-loop and tree-level are proportional, therefore the result of this factorization should also be captured by the tree-level massless five-point amplitude at the correct $\alpha^{\prime}$ order. This is given by the $A_{F^{4}}$ superfield above. As discussed in [32], the factorization in the (12)-channel ((23)-channel) is given by $L_{12} / \alpha_{12}$ $\left(K_{23} / \alpha_{23}\right)$, where

$$
\begin{aligned}
L_{12}= & -40\left[A_{p}^{1}\left(\lambda \gamma^{p} W^{2}\right)+\left(\lambda A^{1}\right)\left(k^{1} \cdot A^{2}\right)\right]\left(\lambda \gamma^{m} W^{5}\right)\left(\lambda \gamma^{n} W^{3}\right) \mathcal{F}_{m n}^{4} \\
& +20\left(k^{1} \cdot k^{2}\right)\left(A^{1} \gamma_{m n} W^{4}\right)\left(\lambda A^{2}\right)\left(\lambda \gamma^{m} W^{5}\right)\left(\lambda \gamma^{n} W^{3}\right) \\
K_{23}= & -40\left[\left(\lambda \gamma^{m} W^{2}\right)\left(k^{2} \cdot A^{3}\right)-\frac{1}{4}\left(\lambda \gamma^{m} \gamma^{r v} W^{3}\right) \mathcal{F}_{r v}^{2}\right]\left(\lambda \gamma^{n} W^{1}\right)\left(\lambda A^{4}\right) \mathcal{F}_{m n}^{5} \\
& +20\left(k^{2} \cdot k^{3}\right)\left(A^{4} \gamma_{m n} W^{5}\right)\left(\lambda A^{3}\right)\left(\lambda \gamma^{m} W^{2}\right)\left(\lambda \gamma^{n} W^{1}\right)-(2 \leftrightarrow 3)
\end{aligned}
$$

Therefore it could be argued that $A_{F^{4}}$ should be proportional to the linear combination $L_{12} / \alpha_{12}+K_{23} / \alpha_{23}+K_{34} / \alpha_{34}+K_{45} / \alpha_{45}+L_{51} / \alpha_{51}$. One can check that the bosonic components satisfy

$$
A_{F^{4}}(\theta)=-\frac{1}{40}\left(\frac{L_{12}}{\alpha_{12}}+\frac{K_{23}}{\alpha_{23}}+\frac{K_{34}}{\alpha_{34}}+\frac{K_{45}}{\alpha_{45}}+\frac{L_{51}}{\alpha_{51}}\right) .
$$

\section{The MHV amplitude}

It is interesting to (formally) rewrite our component expansions in the language of fourdimensional helicity formalism. If the helicities of the gluons are $(--+++)$ we use the following conventions,

$$
e_{\alpha \dot{\alpha}}^{I}=\sqrt{2} \frac{\psi_{\alpha}^{I} \bar{\chi}_{\dot{\alpha}}^{I}}{\left[\bar{\psi}^{I} \bar{\chi}^{I}\right]}, \quad I=1,2, \quad e_{\dot{\beta} \beta}^{J}=\sqrt{2} \frac{\bar{\psi}_{\dot{\beta}}^{J} \chi_{\beta}^{J}}{\left\langle\chi^{J} \psi^{J}\right\rangle}, \quad J=3,4,5
$$

where $\langle\psi \chi\rangle=\psi^{\alpha} \chi_{\alpha}=\epsilon^{\alpha \beta} \psi_{\beta} \chi_{\alpha}$ and $[\bar{\psi} \bar{\chi}]=\bar{\psi}_{\dot{\alpha}} \bar{\chi}^{\dot{\alpha}}=\epsilon_{\dot{\alpha} \dot{\beta}} \bar{\psi}_{\dot{\beta}} \bar{\chi}^{\dot{\alpha}}$ are the spinor products and $\langle i j\rangle[i j]=-2 \alpha_{i j}$. For the specific choice of reference momenta $(2,1,1,1,1)$ they imply

$$
\begin{array}{r}
\left(e^{1} \cdot e^{3}\right)=\left(e^{1} \cdot e^{4}\right)=\left(e^{1} \cdot e^{5}\right)=\left(e^{3} \cdot e^{4}\right)=\left(e^{3} \cdot e^{5}\right)=\left(e^{4} \cdot e^{5}\right)=0 \\
\left(k^{2} \cdot e^{1}\right)=\left(k^{1} \cdot e^{2}\right)=\left(k^{1} \cdot e^{3}\right)=\left(k^{1} \cdot e^{4}\right)=\left(k^{1} \cdot e^{5}\right)=0
\end{array}
$$

and one can check [26] that $L_{12}=L_{51}=L_{2131}=L_{2134}=L_{2314}=0$. With this gauge choice the superfields (2.8) and (2.9) become

$$
\begin{aligned}
& A_{\mathrm{YM}}(\theta)=-\frac{L_{3424}}{\alpha_{34} \alpha_{51}}-\frac{L_{2334}}{\alpha_{23} \alpha_{51}}-\frac{L_{2331}}{\alpha_{23} \alpha_{45}} \\
& A_{F^{4}}(\theta)=-\frac{1}{40}\left[\frac{K_{23}}{\alpha_{23}}+\frac{K_{34}}{\alpha_{34}}+\frac{K_{45}}{\alpha_{45}}\right]
\end{aligned}
$$


where

$$
\begin{aligned}
\left(L_{2331}, L_{3424}, L_{2334}\right) & =-\frac{\sqrt{2}\langle 12\rangle^{4}}{23040 \alpha_{12}}\left(\frac{[23]^{2}[45]}{\langle 14\rangle\langle 15\rangle}, \frac{[25]^{2}[34]}{\langle 13\rangle\langle 14\rangle},-\frac{[23][24][45]}{\langle 13\rangle\langle 14\rangle}\right) \\
K_{23} & =+\frac{\sqrt{2}}{576} \frac{\langle 12\rangle^{3}[23][45]^{2}}{\langle 13\rangle}=-\frac{\sqrt{2}}{72} \alpha_{23} \alpha_{45}\langle 12\rangle^{4}\left[\frac{\alpha_{51}}{N(12345)}+\frac{\alpha_{25}}{N(12543)}\right] \\
K_{34} & =+\frac{\sqrt{2}}{576} \frac{\langle 12\rangle^{4}[25]^{2}[34]}{\langle 13\rangle\langle 14\rangle}=-\frac{\sqrt{2}}{72} \alpha_{25} \alpha_{34}\langle 12\rangle^{4}\left[\frac{\alpha_{35}}{N(12534)}-\frac{\alpha_{45}}{N(12543)}\right] \\
K_{45} & =+\frac{\sqrt{2}}{576} \frac{\langle 12\rangle^{4}[23]^{2}[45]}{\langle 14\rangle\langle 15\rangle}=-\frac{\sqrt{2}}{72} \alpha_{23} \alpha_{45}\langle 12\rangle^{4}\left[\frac{\alpha_{34}}{N(12345)}-\frac{\alpha_{35}}{N(12354)}\right],
\end{aligned}
$$

where $N(i j k l m)=\langle i j\rangle\langle j k\rangle\langle k l\rangle\langle l m\rangle\langle m i\rangle$. Using the results above it is straightforward to obtain, in the NS sector,

$$
A_{\mathrm{YM}}=\mathcal{M}_{\mathrm{MHV}}=\frac{\sqrt{2}}{2880} \frac{\langle 12\rangle^{4}}{\langle 12\rangle\langle 23\rangle\langle 34\rangle\langle 45\rangle\langle 51\rangle},
$$

which agrees with the well-known MHV amplitude up to an overall coefficient. The superfield expression for $A_{F^{4}}$ becomes

$$
A_{F^{4}}=\mathcal{M}_{\mathrm{MHV}}\left[\alpha_{45} \alpha_{51}+\alpha_{23} \alpha_{34}+\alpha_{25} \alpha_{35} \frac{N(12345)}{N(12534)}-\alpha_{23} \alpha_{35} \frac{N(12345)}{N(12354)}\right],
$$

which can be rewritten as

$$
A_{F^{4}}=\mathcal{M}_{\mathrm{MHV}}\left[\alpha_{45} \alpha_{51}+\alpha_{23} \alpha_{34}-[12]\langle 23\rangle[35]\langle 51\rangle\right],
$$

agreeing with (5.45) of [25] and (37) of [23], apart from the overall coefficient.

\section{The integrals $K_{j}$}

In $[17,18]$ the following identities were derived ${ }^{6}$

$$
\begin{aligned}
\alpha_{34} K_{2} & =\alpha_{13} K_{1}+\alpha_{23} K_{4}, & & \alpha_{24} K_{3}=\alpha_{12} K_{1}-\alpha_{23} K_{5}, \\
K_{1} & =K_{4}-K_{5} & & \\
\alpha_{12} K_{2} & =\alpha_{24} K_{1}^{\prime}+\alpha_{23} K_{4}^{\prime}, & & \alpha_{13} K_{3}=\alpha_{34} K_{1}^{\prime}-\alpha_{23} K_{5}^{\prime}, \\
K_{1}^{\prime} & =K_{4}^{\prime}-K_{5}^{\prime} & & \\
\left(1+\alpha_{23}\right) K_{6} & =\alpha_{34} K_{4}^{\prime}-\alpha_{13} K_{5}=\alpha_{12} K_{4}-\alpha_{24} K_{5}^{\prime}, & &
\end{aligned}
$$

and their explicit expansions in terms of $\alpha_{i j}$ were computed at length. However, as mentioned in [18], by defining

$$
T=\alpha_{12} \alpha_{34} K_{2}+\left(\alpha_{12} \alpha_{51}-\alpha_{12} \alpha_{34}+\alpha_{34} \alpha_{45}\right) K_{3},
$$

\footnotetext{
${ }^{6}$ We use a different convention where $\partial_{m}=k_{m}$ instead of $\partial_{m}=i k_{m}$. Therefore one must replace $\alpha_{i j} \rightarrow-\alpha_{i j}$ in the identities of [17]. The only place where it matters is the identity involving $K_{6}$.
} 
all integrals $K_{j}$ and $K_{j}^{\prime}$ can be written in terms of $T$ and $K_{3}$. For example, from (D.1) one can check that (and similarly for $K_{j}^{\prime}$ )

$$
\begin{aligned}
& K_{1}=\frac{\alpha_{34}}{\alpha_{45}} K_{2}+\frac{\alpha_{24}}{\alpha_{45}} K_{3}, \\
& K_{4}=\frac{\alpha_{12} \alpha_{34} K_{2}+\alpha_{23} \alpha_{34} K_{2}-\alpha_{13} \alpha_{24} K_{3}}{\alpha_{23} \alpha_{45}}, K_{5}=\frac{\alpha_{12} \alpha_{34} K_{2}-\alpha_{13} \alpha_{24} K_{3}-\alpha_{23} \alpha_{24} K_{3}}{\alpha_{23} \alpha_{45}}
\end{aligned}
$$

which imply

$$
\begin{aligned}
K_{1} & =\frac{T}{\alpha_{12} \alpha_{45}}-\left(\frac{\alpha_{34}}{\alpha_{12}}+\frac{\alpha_{23}}{\alpha_{45}}\right) K_{3} \\
K_{4} & =\left(\frac{1}{\alpha_{23}}+\frac{1}{\alpha_{12}}\right) \frac{T}{\alpha_{45}}-\left(\frac{\alpha_{51}}{\alpha_{23}}+\frac{\alpha_{34}}{\alpha_{12}}-\frac{\alpha_{13}}{\alpha_{45}}\right) K_{3} \\
K_{5} & =\frac{T}{\alpha_{23} \alpha_{45}}-\left(\frac{\alpha_{12}}{\alpha_{45}}+\frac{\alpha_{51}}{\alpha_{23}}-1\right) K_{3} \\
\left(\alpha_{23}+1\right) K_{6} & =\left(1-\frac{\alpha_{24}}{\alpha_{51}}-\frac{\alpha_{13}}{\alpha_{45}}\right) \frac{T}{\alpha_{23}}+\left(\alpha_{13} \alpha_{24}-\alpha_{12} \alpha_{34}-\alpha_{23} \alpha_{34}-\alpha_{12} \alpha_{23}\right) \frac{K_{3}}{\alpha_{23}}
\end{aligned}
$$

where we used [18],

$$
\begin{array}{ll}
\alpha_{13}=\alpha_{45}-\alpha_{12}-\alpha_{23}, & \alpha_{14}=\alpha_{23}-\alpha_{51}-\alpha_{45}, \\
\alpha_{25}=\alpha_{34}-\alpha_{12}-\alpha_{51}, & \alpha_{35}=\alpha_{12}-\alpha_{45}-\alpha_{34} .
\end{array}
$$

It was shown in [17] that under the twist $\alpha_{12} \leftrightarrow \alpha_{34}, \alpha_{13} \leftrightarrow \alpha_{24}, \alpha_{23} \leftrightarrow \alpha_{23}$ the integrals behave as

$$
\left(T, K_{1}, K_{2}, K_{3}, K_{4}, K_{5}, K_{6}\right) \leftrightarrow\left(T, K_{1}^{\prime}, K_{2}, K_{3}, K_{4}^{\prime}, K_{5}^{\prime}, K_{6}\right)
$$

which allows one to easily obtain $K_{1}^{\prime}, K_{4}^{\prime}$ and $K_{5}^{\prime}$ from (D.3), (D.4) and (D.5).

Open Access. This article is distributed under the terms of the Creative Commons Attribution Noncommercial License which permits any noncommercial use, distribution, and reproduction in any medium, provided the original author(s) and source are credited.

\section{References}

[1] N. Berkovits, Super-Poincaré covariant quantization of the superstring, JHEP 04 (2000) 018 [hep-th/0001035] [SPIRES].

[2] N. Berkovits, Super-Poincaré covariant two-loop superstring amplitudes, JHEP 01 (2006) 005 [hep-th/0503197] [SPIRES].

[3] N. Berkovits and C.R. Mafra, Equivalence of two-loop superstring amplitudes in the pure spinor and RNS formalisms, Phys. Rev. Lett. 96 (2006) 011602 [hep-th/0509234] [SPIRES].

[4] N. Berkovits, Multiloop amplitudes and vanishing theorems using the pure spinor formalism for the superstring, JHEP 09 (2004) 047 [hep-th/0406055] [SPIRES].

[5] C.R. Mafra, Four-point one-loop amplitude computation in the pure spinor formalism, JHEP 01 (2006) 075 [hep-th/0512052] [SPIRES]. 
[6] C.R. Mafra, Pure spinor superspace identities for massless four-point kinematic factors, JHEP 04 (2008) 093 [arXiv:0801.0580] [SPIRES].

[7] N. Berkovits, New higher-derivative $R^{4}$ theorems, Phys. Rev. Lett. 98 (2007) 211601 [hep-th/0609006] [SPIRES].

[8] N. Berkovits, M.B. Green, J.G. Russo and P. Vanhove, Non-renormalization conditions for four-gluon scattering in supersymmetric string and field theory, JHEP 11 (2009) 063 [arXiv:0908.1923] [SPIRES].

[9] M.B. Green, J.G. Russo and P. Vanhove, Ultraviolet properties of maximal supergravity, Phys. Rev. Lett. 98 (2007) 131602 [hep-th/0611273] [SPIRES].

[10] N. Berkovits and N. Nekrasov, Multiloop superstring amplitudes from non-minimal pure spinor formalism, JHEP 12 (2006) 029 [hep-th/0609012] [SPIRES].

[11] Y. Aisaka and N. Berkovits, Pure spinor vertex operators in Siegel gauge and loop amplitude regularization, JHEP 07 (2009) 062 [arXiv: 0903.3443] [SPIRES].

[12] P.A. Grassi and P. Vanhove, Higher-loop amplitudes in the non-minimal pure spinor formalism, JHEP 05 (2009) 089 [arXiv:0903.3903] [SPIRES].

[13] N. Berkovits and B.C. Vallilo, Consistency of super-Poincaré covariant superstring tree amplitudes, JHEP 07 (2000) 015 [hep-th/0004171] [SPIRES].

[14] C.R. Mafra, Superstring scattering amplitudes with the pure spinor formalism, arXiv:0902.1552 [SPIRES].

[15] N. Berkovits, Explaining pure spinor superspace, hep-th/0612021 [SPIRES].

[16] G. Policastro and D. Tsimpis, $R^{4}$, purified, Class. Quant. Grav. 23 (2006) 4753 [hep-th/0603165] [SPIRES].

[17] R. Medina, F.T. Brandt and F.R. Machado, The open superstring 5-point amplitude revisited, JHEP 07 (2002) 071 [hep-th/0208121] [SPIRES].

[18] L.A. Barreiro and R. Medina, 5-field terms in the open superstring effective action, JHEP 03 (2005) 055 [hep-th/0503182] [SPIRES].

[19] Z. Bern, J.J.M. Carrasco and H. Johansson, New relations for gauge-theory amplitudes, Phys. Rev. D 78 (2008) 085011 [arXiv:0805.3993] [SPIRES].

[20] N.E.J. Bjerrum-Bohr, P.H. Damgaard and P. Vanhove, Minimal basis for gauge theory amplitudes, Phys. Rev. Lett. 103 (2009) 161602 [arXiv:0907.1425] [SPIRES].

[21] S. Stieberger, Open \& closed vs. pure open string disk amplitudes, arXiv:0907.2211 [SPIRES].

[22] S. Stieberger and T.R. Taylor, Amplitude for $N$-gluon superstring scattering, Phys. Rev. Lett. 97 (2006) 211601 [hep-th/0607184] [SPIRES].

[23] S. Stieberger and T.R. Taylor, Multi-gluon scattering in open superstring theory, Phys. Rev. D 74 (2006) 126007 [hep-th/0609175] [SPIRES].

[24] S. Stieberger and T.R. Taylor, Supersymmetry relations and MHV amplitudes in superstring theory, Nucl. Phys. B 793 (2008) 83 [arXiv:0708.0574] [SPIRES].

[25] R. Boels, K.J. Larsen, N.A. Obers and M. Vonk, $M H V$, CSW and BCFW: field theory structures in string theory amplitudes, JHEP 11 (2008) 015 [arXiv:0808.2598] [SPIRES]. 
[26] C.R. Mafra, PSS: A FORM program to compute pure spinor superspace expressions, work in progress.

[27] J.A.M. Vermaseren, New features of FORM, math-ph/0010025 [SPIRES].

[28] M. Tentyukov and J.A.M. Vermaseren, The multithreaded version of FORM, hep-ph/0702279 [SPIRES].

[29] N. Berkovits and C.R. Mafra, Some superstring amplitude computations with the non-minimal pure spinor formalism, JHEP 11 (2006) 079 [hep-th/0607187] [SPIRES].

[30] C. Stahn, Fermionic superstring loop amplitudes in the pure spinor formalism, JHEP 05 (2007) 034 [arXiv: 0704.0015] [SPIRES].

[31] R. Medina and L.A. Barreiro, Higher N-point amplitudes in open superstring theory, PoS IC2006 (2006) 038 [hep-th/0611349] [SPIRES].

[32] C.R. Mafra and C. Stahn, The one-loop open superstring massless five-point amplitude with the non-minimal pure spinor formalism, JHEP 03 (2009) 126 [arXiv:0902.1539] [SPIRES]. 